\title{
El impacto del ordenador en la organización de la escuela: perspectivas para la investigación
}

\section{Denis Newman}

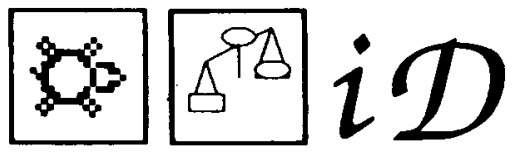

No puede bablarse en abstracto de las virtudes o insuficiencias del ordenador con «el niño» o «la educación». La investigación y experimentación educativa actuales se dirigen a ver cuántos ordenadores $y$ en qué contextos y para qué actividades interactían en el proceso educativo.

El concepto de «apropiación» que se deriva de la teoría sociobistórica subraya que las escuelas pueden aprovechar la tecnología para objetivos muy distintos de los previstos por el investigador.

Los investigadores se interesan por el impacto de los ordenadores en la vida social de las aulas desde los primeras épocas de la introducción de los microordenadores (Hawkins, Sheingold, Gearhart y Berger, 1982; Levin y Kareev, 1980). La Oficina de Evaluación Tecnológica (1988) informa, sin embargo, que a pesar de lo que parecían compras masivas de ordenadores por las escuelas, la relación alumnos-ordenadores está todavía de 30:1 como promedio, lo que significa que el ordenador es poco más que una distracción ocasional en la rutina del aula. Con un solo ordenador por clase, o con un pequeño laboratorio de ordenadores en la escuela, los profesores suelen incorporar la tecnología en su quehacer normal (Cuban, 1986), pero las perspectivas de cambios revolucionarios en la escuela como consecuencia de este nivel de tecnología no son brillantes (Cohen, 1988).

Hay tres factores ahora que ofrecen a los investigadores la oportunidad de volver a estudiar el impacto sobre la organización producido por los ordenadores, es decir, de observar cómo la tecnología reorganiza las interacciones en el aula, cómo da ocasión para una colaboracón entre profesores, y cómo proporciona nuevos modelos de enseñanza.

El primer factor es la compra continua de ordenadores. Si la compra de material tecnológico continúa en los próximos cinco años al mismo ritmo que en los últimos cinco, en muchas escuelas la relación alumnos-ordenadores empezará a convertir el uso del ordenador en una práctica corriente dentro del aula 
en una buena parte del currículum. Habrá escuelas con una relación 4:1, por ejemplo, un número suficiente para permitir hacer generalizaciones acerca del impacto en la organización.

El segundo factor es el desarrollo de un marco teórico basado en los trabajos de Vygotski $(1978,1986)$, que considera el ambiente social como una parte esencial del proceso del cambio cognitivo. El marco ofrece a los investigadores en educación una fuente de conocimiento profundo del impacto sobre el aprendizaje de las distintas maneras de organizar la enseñanza. También les da una metodología para el estudio del cambio en los complejos ambientes educacionales mediante experimentos formativos de las tecnologías que apoyan las diferentes formas de organización.

El tercer factor es el avance logrado en los últimos años en sistemas de ordenadores que sirven a una colectividad más que a un solo trabajador. El sistema de Redes de Area Local (llamado «LAN» en EEUU) se emplea cada día más en los sectores de negocios y de investigación como medio de comunicación y de reparto de datos entre microordenadores. La introducción de esta tecnología de trabajo en colaboración con ordenadores en las escuelas presenta problemas acerca del tipo de organización de la enseñanza que se apoyará en el uso de ordenadores.

Para ilustrar el impacto en la organización de la tecnología en la escuela, sirve el ejemplo de un proyecto diseñado para crear un ambiente para investigaciones científicas en el aula, efectuadas por el sistema de colaboración de grupos. Nuestro interés por el uso de la tecnología como mediadora de cambios en la organización tiene su origen en la teoría que vincula el proceso de aprendizaje directamente a los tipos de interacciones sociales que dan forma a la enseñanza. Nuestro interés en la tecnología específica viene del estado actual de su desarrollo; con la densidad creciente de los ordenadores en las escuelas, el avance tecnológico empieza a posibilitar el uso de medios tecnológicos para cambiar la organización. Un resumen de los fundamentos teóricos y de las cuestiones tecnológicas nos lleva a la metodología del ensayo formativo que se describe en los últimos párrafos de este artículo.

\section{INSTRUMENTOS Y CAMBIO - EL ENFOQUE SOCIO-HISTORICO}

Trabajos recientes en el campo de la ciencia cognitiva que estudian cuestiones de pensamiento y aprendizaje fuera de los ambientes de laboratorio o escuela (Hutchins, en prensa; Lave, 1988; Newman, Griffin y Cole, 1989; Resnick, 1987) han despertado interés por las teorías de desarrollo de Vygotski, Bruner y otros, que han tratado de integrar los procesos de desarrollo interior con los artefactos culturales y los procesos sociales exteriores al individuoo (Bruner, 1966; Laboratorio de Cognición Humana Comparativa, 1983; Rogoff y Lave, 1984; Vygotski, 1978, 1986). En lo que se conoce como la escuela socio-histórica, los procesos cognitivos implican la existencia de instrumentos para pensar, como la escritura, por ejemplo, o los ordenadores, que tienen su propio desarrollo histórico. Estos instrumentos pueden ser interıorizados, como es el caso de nuestro conocimiento del lenguaje, o pueden quedar fuera de nosotros, como el uso de los ordenadores. Un concepto que distingue la escuela socio-histórica de otras actitudes ante el desarrollo es el de «apropiación» - una noción introducida por Leont'ev (1981) —. Los alumnos pueden apropiar los instrumentos de la cultura 
sin captar ni comprender el proceso por el cual el instrumento fue creado históricamente. De hecho, un instrumento puede tener una interpretación totalmente distinta para el niño y para el adulto. El mismo objeto puede asumir papeles diferentes en los dos sistemas de actividad.

El sistema cognitivo interpersonal es importante para la comprensión del cambio cognitivo porque (a) los instrumentos elaborados culturalmente juegan un papel crítico, y (b) las actividades en las que actúan los instrumentos se transmiten sólo a través de un aprendizaje. Vygotsky $(1978,1986)$ introdujo el concepto de una zona de desarrollo próximo (ZDP) en la que los niños, ayudados por otros, pueden trabajar en problemas que están más allá de su competencia como individuos. Los conocimientos y las habilidades que son incorporados en su interior, según esta opinión, son los procesos interactivos que se utilizan para abordar el problema. La «apropiación» es un proceso importante en la ZDP, por parte del alumno y del profesor. Newman, Griffin y Cole (1989) describen sesiones de enseñanza y de tutoría en el transcurso de las cuales el profesor apropia las acciones del alumno dentro de su propia manera de entender el trabajo. El profesor ha de encontrar una vía por la que el alumno tenga un papel, aunque sea mínimo, en la ejecución de la tarea, y dar la respuesta en términos de una comprensión experta de la misma: -Cuál es el objetivo, qué es lo relevante, por qué uno de los pasos no dio resultado, etc. Lo único que tiene que hacer el alumno es encontrar un paso que contribuya de alguna manera (o que puede interpretarse como un intento de contribuir) a la tarea. El comprobar lo adecuado de su acción proporciona al alumno un análisis de la tarea tal como la comprende el profesor. De esta forma, el conocimiento se construye activamente en las interacciones sociales en donde el significado de una acción puede ser cambiado retrospectivamente por las acciones posteriores de otras personas (Fox, 1987; Newman y Bruce, 1986).

El concepto de apropiación es aplicable también a la adopción de tecnología en el ambiente educacional. Los instrumentos se interpretan en términos de la estructura actual de las actividades. Los instrumentos pueden servir para aumentar el potencial del actor, pero los ampliadores han de ser entendidos en términos de las capacidades individuales (Cole y Griffin, 1980; Pea, 1985). Los ordenadores amplían la capacidad de un profesor para toda una serie de tipos de enseñanza, no para un cambio en el profesor sino con un cambio de la actividad, un cambio, por ejemplo, de una actividad educativa impartida por el profesor a partir de un libro a un trabajo de simulación efectuado por los alumnos trabajando en pequeños grupos. El objetivo del profesor - de transmitir una comprensión de algún sistema físico- se consigue con más eficacia mediante un cambio en la organización de la enseñanza.

Los cambios que se producen cuando la tecnología es apropiada para el medio ambiente pueden parecer mínimos si el ordenador sólo se encaja en la estructura existente (Cuban, 1986). Por ejemplo, Mehan (1988) describe aulas de grado elemental, organizadas en «centros», que no hacían más que convertir el ordenador del aula en otro centro. La escuela tiene que apropiar la tecnología para hacer uso de ella, pero en algunas condiciones, puede apropiar una tecnología que ayuda, a su vez, a cambiar el ambiente educacional. Este proceso puede tener como resultado una nueva interpretación del ordenador, así como un cambio constructivo de la actividad en el aula (Bruce y Rubin, en prensa). Las nuevas tecnologías, ideadas para facilitar nuevas formas de organización social, representan un desafío interesante para las escuelas. 


\section{EL TRABAJO EN GRUPOS APOYADO POR EL ORDENADOR}

El uso de ordenadores en los laboratorios de investigación y en oficinas comerciales supone una tecnología diseñada para facilitar la organización del centro de trabajo. El hecho de que exista un sector de investigación y desarrollo dedicado a la organización de ambientes informatizados es otro motivo para explorar posibles caminos paralelos en el ambiente educacional. En las discusiones sobre el uso de los ordenadores en educación, el término «ambiente» suele significar un programa que permite al estudiante explorar una simulación idealizada de un sistema real -físico o matemático-. En cambio, el término «ambiente educacional» se aplica al mundo social en donde funciona cualquiera de las tecnologías. El ambiente total puede simular un entorno social real -un laboratorio de investigación, por ejemplo, o una fábrica-, utilizando la tecnología de forma análoga. Las investigaciones sobre el papel de la tecnología en la construcción de tales simulaciones puede servirse de trabajos prácticos para la creación de ambiente reales.

En los últimos años, los investigadores y expertos en desarrollo han establecido un nuevo terreno dedicado a lo que se llama «trabajo en colaboración apoyado en los ordenadores» (1986, 1988; Galegher, Kraut y Egido, en prensa). El terreno comprende trabajos sobre «correo electrónico», conferencias por vídeo, bancos de datos distribuidos, inventarios de grupo, y «collaborative hypertext environments». Por ejemplo, Xerox PARC ha explorado un sistema que facilita la investigación y el reportaje en colaboración mediante un sistema hypertext (Trigg, Suchman, y Halasz, 1986). La integración de la comunicación escrita con la estructura de notas de investigación ofrece un modelo importante para la colaboración mediante Redes Locales. Los investigadore de Xerox proyectan un sistema de apoyo a las reuniones personales de los diseñadores (Stefic, Foster, Bobrow, Kahn, Lanning y Suchman, 1987). Los trabajos en esta área van más allá, naturalmente, de las pruebas del interface persona-máquina, puesto que la tecnología está involucrada en la organización social. Por ejemplo, Engestrom, Engestrom y Saalrema (1988) describen los cambios en el trabajo de los médicos en un hospital en donde los historiales de los enfermos se convierten en un recurso compartido a través de los ordenadores. Como escribe Grudin (en prensa), debido a la falta de análisis de los cambios y conflictos sociales, muchas aplicaciones para trabajos en colaboración fracasan porque los diseñadores tienen ideas simplistas sobre cuestiones de organización, e ignoran muchas veces las incomodidades que han de sufrir los subordinados para que los supervisores estén bien.

En el mundo de los adultos, los ordenadores suelen emplearse en el contexto de trabajo donde el software sirve para crear, analizar y comunicar información. El uso en las escuelas es muy distinto, sobre todo en lo que se refiere a la tecnología de redes - el núcleo de muchas aplicaciones de colaboración por ordenadores-. Aquí el enlace de los ordenadores en la red sirve de solución fácil para la entrega de software a los ordenadores individuales. En las escuelas, muchas veces el uso se basa en programas más que en información como es el caso en las oficinas. Esta orientación se aprecia claramente en situaciones como los sistemas integrados de aprendizaje (Oficina de Valoración de la Tecnología, 1988) que ofrecen programas de instrucción por secuencias progresivas para extensas áreas del currículum, dejando a los profesores el papel de controlador y monitor. Del mismo modo, los sistemas de Red Local que se venden a las es- 
cuelas ofrecen a los alumnos un menú de programas entre los que puede elegir, y donde el profesor tiene pocas posibilidades de acceder a una serie determinada. En cambio, en el mundo de los adultos, es más importante tener acceso a bloques de información que a una amplia variedad de programas.

El nuevo campo de trabajo en colaboración mediante una red de ordenadores ofrece por un lado una tecnología con posible aplicación en las escuelas, y por otro lado una base de investigación que valora negativamente esta tecnología en téminos de la organización del ambiente de trabajo. Muchas veces se supone que cualquier trabajo con ordenadores implica una organización social; incluso cuando las relaciones jerárquicas no sean muy cooperativas (Bannon y Schmidt, 1989). La investigación de este sistema de trabajo en colaboración está ayudando, pues, a formar la idea de que la tecnología es una parte de la actividad laboral organizada socialmente. Tienen especial importancia los estudios, cada vez más numerosos, que analizan el aprendizaje y los cambios de organización como consecuencia de la introducción de la tecnología.

\section{EL AMBIENTE EDUCACIONAL - ENSAYOS FORMATIVOS}

El continuo aumento del número de ordenadores en las escuelas significa una creciente preocupación por parte de los educadores acerca del impacto que tendrán en la organización en las aulas. Los modelos propuestos para la solución técnica de esta reorganización, así como una teoría de cambio cognitivo que incluye la organización social de la enseñanza, hacen de la experimentación, además, una cuestión práctica para los investigadores. La tecnología para apoyar la organización social se combina con una teoría en la que el aprendizaje es un proceso social, instrumentado por material tecnológico; esto sugiere un planteamiento metodológico para el uso de la tecnología en la enseñanza actual.

Un experimento formativo puede necesitar planes complejos de preparación de profesores, de desarrollo de planes de estudio, y de producción de materiales para las aulas para crear un ambiente para la confrontación de las tareas por parte de los alumnos y de los profesores. Es fundamental que el trabajo se haga en las aulas, no en los laboratorios, puesto que se trata de observar el impacto en la organización. Pero un experimento formativo, en el sentido que empleamos aquí, proporciona otras ventajas que no ofrece la experimentación tradicional. En un experimento formativo, el investigador establece un objetivo pedagógico, y descubre lo que requiere en términos de material, organización, o modificaciones de la tecnología para conseguir el objetivo. En vez de controlar estrictamente los tratamientos y de observar las diferencias en el resultado, como es el caso en un ensayo convencional, los experimentos formativos apuntan a un resultado específico y luego observan el proceso por el que se consigue este resultado (P. Griffin, comunicación personal). Este formato es el que se emplea en la evaluación formativa del software (Hawkins y Kurland, 1987); el diseñador mejora el producto con repetidas pruebas hasta que resulte satisfactorio, tanto por su atractivo como por su efectividad. Si es el ambiente, más que la tecnología, el tema de análisis, entonces se observan los cambios en las interacciones durante la enseñanza, los cambios en el papel del profesor y cualquier cambio en el ambiente educacional. El formato del experimento formativo, en una intervención dentro del aula o en toda la escuela, sirve para estudiar las interacciones entre los alumnos y los profesores, tanto a nivel de pequeños grupos como en las cuestiones más amplias de la reestructuración. Los cambios en 


\section{8}

la organización provocados por el soporte tecnológico pueden entenderse también como cambios en el soporte para la cognición, es decir, como nuevas zonas de desarrollo próximo que aumentan las posibilidades para las interacciones educativas.

Los distintos objetivos pedagógicos nos proponen nuevos planteamientos para el uso en gran escala de los ordenadores y requerirán distintos tipos de soporte, tecnológicos y otros. Una pedagogía que pone el énfasis en la adquisición de habilidades básicas sugiere el uso de un sistema de aprendizaje integrado en que las prácticas y ejercicios sean individualizados y progresivos. Según Cole y Griffin (1987), tales sitemas se encuentran cada vez más en las escuelas que atienden a poblaciones más pobres en donde la preocupación por los resultados de los exámenes motiva la compra de ordenadores.

Por el contrario, una pedagogía que ve el aprendizaje como una manera de llegar a capacidades cognitivas y comprensión más altas (Collins, Brown y Newman, 1989; Resnick, 1987) sugiere una utilización de los ordenadores en la escuela siguiendo el modelo de las actividades laborales de los adultos. Cada idea de la educación incluye una imagen única de cómo funcionan los ordenadores en la organización del aula. Para crear los distintos ambientes que se desean, necesitaremos diferentes tipos de soporte. En sus experimentos formativos en busca de un objetivo específico, los investigadores pueden informar sobre el nivel de esfuerzo necesario para conseguirlo.

Esta noción de un experimento formativo se parece a la forma en que muchas veces se emplea el concepto de zona de desarrollo ZDP próximo en aquellos exámenes en los que la cantidad de ayuda que necesita el sujeto determina su puesto dentro de la zona. El uso de un punto determinado que hay que alcanzar («static end point»), aunque sea un componente necesario de los tests, no es, sin embargo, inherente a la teoría de zona de desarrollo próximo (Campione, Brown, Ferrara y Bryant, 1984). En el estudio del proceso de cambio cognitivo mediante observaciones de las interacciones de la zona de desarrollo próximo, es de igual importancia entender dónde los aprendices pueden obtener ayuda. No todos llegarán tan lejos, ni todos irán en la misma dirección; son muchos los puntos y destinos, puesto que el alumno es también un apropiador activo de los instrumentos ofrecidos por el profesor. Es decir, los alumnos utilizan los instrumentos de la cultura para la comprensión de sus propias tareas, reorganizando y ampliando de esta forma sus propias capacidades.

Cualquiera que sea la teoría pedagógica que motive el experimento, el resultado que ha de ser observado debe incluir cómo cambia la organización del ambiente a medida que apropie la tecnología y los otros recursos. El ambiente puede superar sus objetivos iniciales. También puede retener los objetivos y la organización a pesar de los esfuerzos del diseñador de la tecnología por apoyar modelos alternativos. No será posible imponer a las escuelas un modelo completamente ajeno al uso de los ordenadores. Por ejemplo, el modelo de una oficina equipada con alta tecnología no es transferible a las escuelas ni siquiera si se dispusiera de la misma tecnología; porque la tarea de aprender no es la de ejecutar funciones conocidas, y puede necesitar distintas formas de interacción con la tecnología, y otro tipo de cooperación con los compañeros. En un experimento formativo, el investigador puede aprender mucho tratando de crear otro tipo de ambiente, diferente en términos de los soportes necesarios y también en términos de la apropiación de estos soportes por el ambiente para ir en direcciones imprevisibles.

El estudio que se describe en la siguiente sección explica los cambios desa- 
rrollados en un ambiente educacional tradicional después de la introducción de la tecnología diseñada para el uso de ordenadores en investigaciones científicas. Las observaciones de los cambios en la organización demuestran cómo un experimento formativo debe descubrir hasta qué punto el ambiente aprovecha la intervención.

\section{UN EXPERIMENTO EN UN AMBIENTE ESCOLAR DE RED LOCAL}

En el transcurso de los últimos cuatro años, el proyecto Earth Lab (Laboratorio de Ciencias de la Tierra) se ha dedicado al diseño, el montaje y la observación de los efectos de un sistema de Red Local de ordenadores cuya función es el fomento del trabajo en colaboración en el tema de ciencias de la Tierra en una escuela primaria. La idea consistió en crear un prototipo de red local de ordenadores, y probarlo en una escuela estatal de Nueva York empleando un currículum de ciencias de la Tierra (Newman y Goldman, 1987). El motivo pedagógico fue que los alumnos usaran la tecnología como lo hacen los científicos: para comunicar y compartir datos, es decir para colaborar. En el proyecto, empleamos tecnología educativa específica para cambiar el ambiente educativo pasando de uno que desaprobaba el trabajo en colaboración entre los alumnos a otro en donde esta colaboración fuera normal. El objetivo del experimento, aumentar la frecuencia de trabajar en colaboración. Estábamos dispuestos a modificar el diseño de la tecnologías, a introducir nuevo software, desarrollar materiales del currículum y a organizar talleres para los profesores de acuerdo con las necesidades.

\section{DESARROLLAR EL AMBIENTE}

El experimento duró un año y terminó en junio, 1987. En una Red Local, se conectaron 25 ordenadores Apple II de la escuela con una central de almacenamiento de datos, de texto y programas. El programa de procesamiento de texto Bank Street se amplió con un sistema de «correo electrónico» (Newman, 1987). Otro componente básico fue el Archivador Bank Street que permitía a los alumnos crear bancos de datos que podrían ser evaluados a partir de cualquier ordenador de la escuela.

Se utilizaron mucho los bancos de datos, dentro y fuera del currículum de ciencias de la Tierra. Durante la hora de la comida, los alumnos inventaban bancos de datos con sus imágenes favoritas. En los períodos de estudios sociales, buscaban en los calendarios y otros sitios para llenar ficheros de datos sobre países y personajes históricos. En Ciencias de la Tierra repasaron datos de fósiles de dinosaurios y de terremotos, y crearon un banco de datos de los fenómenos meteorológicos recogidos por pequeños grupos de estudiantes durante tres meses.

El principal medio de apoyo a los grupos colaboradores fue la administración de los datos recogidos por los grupos e individualmente. Esto fue el papel del interface de la Red del Laboratorio de la Tierra que facilitó el almacenamiento y la recuperación de los datos pertinentes. A los estudiantes y profesores se les asignaban un número indeterminado de ficheros. Por ejemplo, había ficheros para parejas de alumnos que preparaban juntos un escrito. Otros se destinaban a clubs de la escuela, como los Jóvenes Astronautas. Cada individuo tenía su fichero personal. El profesor de ciencias, que tenían dos clases por se- 
mana, dividió la clase en grupos de tres o cuatro para hacer investigaciones en el aula de ciencias. Estos grupos se asignaron nombres con los que se identificaban en la Red, y todos compartían datos con los otros alumnos o grupos en la escuela. Podía ser que un alumno compartiese datos con un grupo de ciencias, con un club de la hora de comer, y con la clase entera. Habría sido imposible que cada uno tuviese su propio disquete, ya que la organización social del acceso a los datos exigía un gran número de vías de acceso al banco de datos.

El Laboratorio de la Tierra era único entre las redes escolares, único en el sentido de que su orientación era hacia los datos, no hacia programas. El interface presentaba listas de proyectos y de grupos de trabajo a elegir, y sólo después, una lista de programas con los que se podía trabajar sobre los datos. También fue diseñado esencialmente para la colaboración; los datos estaban accesibles a los grupos, y con el procesador de textos era tan fácil trasladar un fichero a otro participante como grabarlo para uso de un grupo o individuo.

Junto con la tecnología, presentamos un currículum de ciencias de la tierra, de un año de duración, preparado en colaboración con los profesores (Brienne y Goldman, 1989). Muchas de las actividades se idearon para la comunicación y colaboración entre los alumnos, con la intención de que fueran utilizadas. Lo que observamos fue más y al mismo tiempo menos de lo que habíamos esperado. No hubo ninguna transformación radical de la escuela; la innovación hizo efecto poco a poco. En algunas áreas, la forma de usar el sistema superó nuestras esperanzas iniciales. Los ejemplos que pasamos a detallar no pretenden demostrar cuantitativamente los cambios, sino ilustrar los mecanismos por medio de los cuales pueden ocurrir cambios con esta tecnología.

\section{MEDIACION DE LA COLABORACION DEL PROFESOR}

Durante el año, no se observó ningún cambio importante respecto al de pequeños grupos de trabajo. Encontramos que los grupos que se formaban en el aula de ciencias fueron utilizados por los profesores para una serie de actividades relacionadas con estudios sociales, que en algunos casos no tenían nada que ver con el currículum de Ciencias de la Tierra. El sistema de la Red produjo cambios de una manera inesperada: hizo posible que todos los profesores asignaran trabajos de clase a los grupos creados por el profesor de ciencias. Los ficheros de estos grupos representaban un medio fácil para la organización de proyectos de grupo en otras áreas del currículum. Los grupos de ciencias llegaron a ser un recurso para toda la escuela. Nunca había habido un mecanismo mediante el cual una estructura de organización social, creada por uno de los profesores, en esta escuela pudiese ser aprovechada por los otros profesores como recurso didáctico (Newman, Goldman, Brienne, Jackson y Magzamen, 1989).

\section{COORDINACION DE LAS INVESTIGACIONES CIENTIFICAS DE LOS ALUMNOS}

Al establecerse un banco de datos compartido, nuestra aplicación de la tecnología de Red Local parecía, naturalmente, una invitación a la colaboración, en la que los alumnos contribuyen a una búsqueda de conocimientos más amplia porque era más fácil dar acceso común al mismo banco de datos que mantener copias individuales por cada alumno o grupo (Newman et al., 1989). Un ejemplo puede ser nuestro proyecto de recogida de datos climatológicos. Cada 
pequeño grupo por turno recogía los datos, formando un banco compartido que se utilizó después para averiguar las correlaciones entre las variables, tales como la presión atmosférica y la nubosidad. Toda la serie de datos fueron puestos en común por el grupo como objeto de discusión que de otra manera no hubieran sabido encontrar, contribuyendo individualmente. Esta coordinación alrededor de un banco de datos compartido fue una nueva estructura de actividad que surgió con la tecnología de Redes Locales.

\section{EL TRANSPORTE DE TAREAS DE UN SITIO A OTRO}

Habíamos anticipado que los alumnos empezarían un trabajo mientras la clase estuviese en el laboratorio de ordenadores para continuarlo en el aula. De hecho, esta flexibilidad se llevó más lejos. Vimos a dos alumnos que trabajaban juntos en unos resúmenes o informes de un libro, con un ordenador de su aula. No habían terminado cuando tocó el turno de su clase de pasar al laboratorio de ordenadores. En vez de dejar su tarea, se fueron con los apuntes y pidieron permiso para trabajar en uno de los ordenadores del laboratorio mientras los demás trabajaban en otra asignatura. Encontraron el fichero en que habían trabajado. Las «fronteras» entre una clase y otra ya parecían permeables, y esta permeabilidad fue empleada por los alumnos para trabajar por iniciativa propia.

\section{USO DE LA RED PARA TRABAJOS PERSONALES DE LOS ALUMNOS}

Muchos alumnos usaron el sistema para sus propios trabajos además de utilizarlo para las tareas asignadas en clase. Su sentido de propiedad en cuanto a sus ficheros nos sorprendió. Algunos acumularon cientos de fichas personales en el curso del año. El proyecto Laboratorio de la Tierra fue aprovechado también para alumnos iniciados en trabajos en equipo tales como proyectos científicos para «exposición» - (para los padres y visitantes a final del curso)-, un orgullo para muchos grupos de estudiantes. Su apropiación de la tecnología se hizo evidente también en las entrevistas a final del curso. Uno de los alumnos, por ejemplo, propuso que hubiera ficheros para que los alumnos determinaran prioridades de acceso - que fuera fácil entrar en el trabajo que un alumno hacía con otro-. Fue un invento basado en una necesidad que surgió de los intentos personales de actuar en colaboración.

\section{USO DEL «CORREO ELECTRONICO»}

El sistema de comunicación que se incorporó en el procesador de textos fue ampliamente usado para comunicación tanto académica como extra-curricular (Goldman y Newman, en prensa). En el currículum de ciencias de la Tierra, desarrollamos actividades en las que los grupos trabajan sobre una cuestión semanal, comunicando sus respuestas por el «correo», y también mandaban preguntas acerca de sus estudios de ciencias a otros grupos. Aparte de estas actividades estructuradas, el sistema de comunicación abrió otras nuevos canales. Por ejemplo, entre los alumnos y el profesor se mantenían conversaciones individuales, algo que no era frecuente en el aula normal. Muchos alumnos usaban el sistema para comunicación privada y algunos mantenían grandes intercambios con otras personas, incluidos adultos a través del enlace de telecomunicación (Goldman y Chaiklin, en preparación). 


\section{RESUMEN Y FUTUROS PASOS}

En todas estas observaciones se aprecia el hecho de que los alumnos tenían su sitio en el sistema informático, es decir, su fichero con sus datos para el proyecto, fichero que podía ser individual o de un grupo y que esto no dependía de que tuviese cada uno un ordenador ni que se encontraran en un aula determinada. Tanto los alumnos como los profesores aprovecharon los ficheros como puente entre diferentes ambientes escolares. El grado de apropiación del siste$\mathrm{ma}$ - tanto por los profesores como por los alumnos- en su propio trabajo parece indicar que los sistemas del tipo de Laboratorio de la Tierra son válidos en el ambiente educacional. El sistema que instalamos en la escuela se diseñó, hasta cierto punto, basado en la tecnología empleada en los laboratorios de investigación, pero el sistema que surgió tenía características totalmente específicas para las escuelas. Por ejemplo, la coordinación de pequeños grupos, la colaboración del profesor, y el uso de ficheros (espacios) en vez de equipos personales. Nuestro experimento formativo logró usar la tecnología para facilitar el trabajo en grupos pero descubrimos también que una función importante de la tecnología de Red Local en las escuelas consiste en establecer una mayor interrelación entre los departamentos.

La próxima etapa de nuestro trabajo en Redes Locales para las escuelas será una reformulación del objetivo de la tecnología: en vez de concentrar nuestros esfuerzos en la extensión de la colaboración, pensamos extender el uso de proyectos que eliminen la actual compartimentación en el currículum. Tales proyectos, sean individuales o colectivos, pueden romper la departamentalización general de la escuela, dando a los alumnos el sentido de ser aceptados - de pertenecer- en las actividades educativas, con independencia de las tareas impuestas por los profesores. En apoyo de este planteamiento, será útil crear instrumentos que ayuden a los alumnos a organizar y coordinar sus investigaciones (Newman, 1988). Sospechamos que la solución a los problemas que tienen los profesores para organizar la enseñanza de manera que implique colaboración entre los alumnos, así como la colaboración con sus colegas, está en dotar a los alumnos de instrumentos que asuman una parte de la carga, en vez de dotar a los profesores de instrumentos que les confieran un mayor control. Los avances futuros en materia de diseño y los nuevos ensayos formativos, mejorarán el modelo y llevarán más lejos los cambios que pueden hacerse en el ambiente escolar.

\section{CONCLUSIONES}

El experimento formativo que se hizo en una escuela proporciona datos para avanzar en el diseño de redes locales de ordenadores para las escuelas. Expone también las características de un tipo de investigación que puede aprovechar la oportunidad para estudiar mediante observación, el impacto de la nueva tecnología en la organización. En segundo lugar, la lógica de un experimento formativo en el que el investigador analiza el soporte que hace falta para conseguir un objetivo incial, ha de permitir un cambio en los objetivos a medida que el ambiente se apropia de la tecnología. La tecnología es tratada no como causa del cambio, sino como algo que puede ser usado, tanto por la escuela como por el investigador, como soporte de cambios.

A medida que la investigación vaya hilando más fino en el análisis de las interacciones educacionales que constituyen estos cambios, el análisis sociohis- 
tórico de la enseñanza en clase (Newman, Griffin y Cope, 1989) proporciona un enlace entre la escuela o el aula como unidad de análisis y la interacción entre el profesor y el alumnos (o entre los alumnos) como unidad de análisis.

Para explorar en qué condiciones funciona mejor un sistema tecnológico, será necesario un proceso de investigación sistemático. Por ejemplo, pueden ser factores importantes para el éxito de un proyecto del tipo de Laboratorio de la Tierra el número de alumnos por ordenador, el apoyo prestado a los profesores y a su preparación, el nivel escolar y el contenido de la eseñanza. Los trabajos emprendidos por el Centro de Tecnología (Bank Street College of Education) y el Instituto de Alfabetización (Education Development Centre) con sus colaboradores en $\mathrm{BBN}$, incluyen una serie de experimentos destinados a averiguar cómo contribuyen los distintos diseños de ambiente escolar en términos de aprendizaje, cooperación, motivación, etc. (Collins, en prensa). Collins afirma que tales investigaciones pueden conducir a una «ciencia del diseño», más parecida a la aeronáutica que a las ciencias naturales, ya que tratará de descubrir aquellos factores que entran en juego ante determinados objetivos, de la misma manera que en la aeronáutica los distintos diseños afectan la elevación, la resistencia al avance, y la maniobrabilidad. Es fundamentalmente una ciencia de lo artificial, como la define Simon (1981).

El Laboratorio de la Tierra contribuye a la ciencia del diseño con varias hipótesis respecto a las variables que pueden entrar en juego: el número de alumnos por ordenador, el grado de movilidad entre las aulas y el laboratorio y las estructuras de las actividades del currículum. La razón de la ciencia del diseño se expresa en el lenguaje de las variables dependientes e independientes, pero no obstante, implica profundamente las cuestiones de la organización de las aulas y el apoyo que se preste al cambio en los experimentos de tipo formativo. En la práctica, son las pequeñas modificaciones y la observación minuciosa de lo que ocurre en la escuela como consecuencia de los medios de soporte, lo que sustituye a la definición de las condiciones del experimento y la especificación detallada de las medidas dependientes.

El estado actual de la ciencia del diseño en la educación hace difícil una especificación a priori de los objetivos de una intervención; no sabemos lo que puede conseguirse por medio de la tecnología, ni sabemos siquiera lo que hará falta en términos de habilidades cognitivas, motivación, o pertenencia a la comunidad para atender a las necesidades futuras de la sociedad. La investigación del impacto de los ordenadores en la organización en las escuelas puede avanzar mediante una definición de los objetivos razonables para la entrada de la tecnología moderna en la escuela, pero al mismo tiempo ha de estar abierta a la aparición de nuevos objetivos. Mediante el análisis de la intervención, podremos comprender mejor el proceso educativo, viendo cómo la tecnología ayuda a sostener las zonas de desarrollo próximo que servirán tanto a los estudiantes como a los profesores en la consecución de sus objetivos educativos. 


\section{Referencias}

Bannon, L. J., y Schmidt, K. (1989, Semptember). CSCW: Four characters in search of a context. Paper presented at the First European Conference on Computer Supported Cooperative Work, London.

Brienne, D., y Goldman, S.V. (1989, April). Networking: How it has enhanced sciencie classes in New York schools... and how it can enhance classes in your school, too. Classroom Computer Learning. p.p. $44-53$

Bruce, B., y RuBin, A. (en prensa). Electronic quills: $A$ situated evaluation of using computers for writing in classrooms. Hillsdale, NJ: Erlbaum.

Bruner, J.S. (1966). On cognitive growth. In J. S. Bruner, R. R. Olver y P.M. Greenfield (Eds.), Studies in cognitive growth. Nueva York: Wiley.

Campione, J.C., Brown, A.L., Ferrara, R., y Bryant, N.R. (1984). The zone of proximal development: Implications for individual differences and learnins. In B. Rogoff y J. V. Wertsch (Eds.), Cbildren's learning in the zone of proximal development: New directions for child development, 2, 45-63. San Francisco, CA: Jossey-Bass.

Cohen, D.K. (1988). Educational technology and school organization. In R. S. Nickerson (Ed.), Technology and education: Looking toward 2020. Hillsdale NJ: Erlbaum.

Cole, M., y Griffin, P. (1980). Cultural amplifiers reconsidered. In D. Olson (Ed.), Social foundations of language and thought. Nueva York: W.W. Norton.

Cole, M., y Grifrin, P. (Eds.), (1987). Contextual factors in education. Madison, WI: University of Wisconsin, Wisconsin Center for Education Research.

Coluins, A. (1989). Toward a design science of education. In E. Scanlon y T. O'Shea (Eds.), Cognition and instruction: Issues and agenda. Hillsdale, NJ: Erlbaum.

Cscw 86: Proceedings of the conference on computer supported cooperative work (1986). Austin, TX: Author.

Cscw 88: Procedings of the conference on computer-supported cooperative work. (1988). Portland, OR: Author.

Cuban, L. (1986). Teachers and machines. Nueva York: Teachers College. Engestrom, y., Engestrom, R., y Saarelma, O. (1988). Computerized medical records, production pressure and compartmentalization in the work activity of health center physicians. In CSCW 88: Proceedings of the Conference en Computer-supported Cooperative Work, September, 1988. Porland, OR: Author.

Fox, B. (1987). Interactional reconstruction in real-time language processing. Cognitive Science, 11(3), 365-387.

Galegher, J., Kraut, R., y EGido, C. (en prensa). Intellectual teamwork: social and technical bases of cooperative work. Hillsdale, $\mathrm{HJ}$ : Erlbaum.

Goldman, S.V., y ChaIKIN, S. (en preparación). Using telecommunications in mentoring relationships with elementary students. Annual Meeting of the American Educational Research Association, Boston, 1990.

Goldman, S.V., y Newman, D. (en prensa). Electronic interactions: How students and teachers organize schooling over the wires. Discourse Processes.

Grudin, J. (en prensa). Why CSCW applications fail: Problems in the design and evaluation of organization inerfaces. Office technology and People.

Hawins, J. Y KuRland, D.M. (1987). Informing the design of software through context-based research. In R. Pea y K. Sheigold (Eds.), Mirrors of minds: Patterns of experience in educational computing. Norwood, NJ: Ablex Publishing Corp.

Hawkins, J., Sheingold, K., Gearhard, M., y Berger, C. (1982). Microcomputers in schools: Impact on the social life of elementary classrooms. Journal of Applied Developmental Psychology, 3, 361-373.

Hutchins, E. (in press). The technology of team navigation. In J. Galegher, R. Kraut, y C. Egido (Eds.), Intellectual teanwork: Social and tecbnical bases of cooperative work. Hillsdale, NJ: Erlbaum.

Laboratory of Comparative human Cognitions. (1983). Culture and cognitive development. In W. Kessen (Ed.), Mussen's handbook of child psycbology: Vol. 1. History, theory, and method (4th ed., pp. 295-356). Nueva York: Wiley.

Lave. J. (1988). Cognition in practice; mind, mathematics and culture in everyday life. Cambridge: Cambridge University Press.

Leontev, A.N. (1981). Problems of the development of mind. Moscú: Progress Publishers.

Levin, J.A., y Kareev, Y. (1980). Personal computers and education: The challenge to schools. La Jolla, CA: Center for Human Information Processing.

Mehan, H. (1988). Microcomputers in classrooms: Educational technology or social practice? $A n$ thropology and Education Quarterly, 20, 5-22.

Newman, D. (1987). Local and long-distance computer networking for science classrooms. Educational Technology, 27 (6), 20-23.

Newman, D. (1988, octubre). Sixth graders and shared data. Designins a LAN environment to support collaborative work. Döcumento presentado a la $2 .^{a}$ Conferencia de Computer Supported Cooperative Work, Portland, OR. 
Newman, D., y Bruce, B.C. (1986). Interpretation and manipulation in human plans. Discourse Processes, 9, 167-195

Newman, D., Goldman, S.V., Brienne, D., Jackson, I., y Magzamen, S. (1989). Peer collaboration in computer-mediated science investigations, Joumal of Educational Computing Research, 5(2), 151-166.

Newman, D., Griffin, P., y Cole, M. (1989). The construction zone: Working for cognitive change in school. Cambridge: Cambridge University Press.

Office of Technology Assessment. (1988). Power on! New tools for teaching and learning (OTA Sep. 379). Washington, DC: U.S. Government Printing Office.

PEA, R.D. (1985). Beyond amplification: Using the computer to reorganize mental functioning. Educational Pychologist, 20, 167-182.

ResNick, L.B. (1987). Learning in school and out. Educational Researcher, 16(9) 13-20.

Rogoff, B., y Lave, J. (Eds.). (1984). Everyday cognition: Its development in social context. Cambridge, MA: Harvard University Press.

Simon, H.A. (1981). The sciences of the artificial (2. ${ }^{\text {a }}$ ed.). Cambridge, MA: MIT Press.

Stefic, M., Foster, G., Bobrow, D.G., Kahn, K., Lanning, S., y Suchman, L. (1987). Beyond the chalkboard: Computer support for collaboration and problem solving in meetings. Communications of the $A C M, 30,32-47$.

Trigg, R. H., Suchman, L.A., y Halasz, F.G. (1986). Supporting collaboration in notecards. Proceedings of the conference on computer-supported cooperative work, Austin, TX.

VYGorski, L.S. (1978). Mind in society: The development of bisher psycbological processes (M. Cole, V. John-Steiner, S. Scribner, y E. Souberman, Eds, and Trans.). Cambridge: Harvard University Press.

VyGoTski, L.S. (1986). Thought and language. (A. Kozulin, Ed. and trans. Cambridge, MA; MIT Press.

\section{El impacto del ordenador}

en la organización de la escuela:

perspectivas para la investigación.

D. Newman.

$C L \& E, 1992,13$, pp. $23-35$

Datos sobre el autor: Denis Newman es miembro científico del BBN Systems and Techno-

logies Corporation.

Dirección: BBN Systems and Technologies Corporation. Cambridge, Massachusetts.

Artículo original: Opportunities for Research on the organizational Impact of School Computers. Educational Researcher, Vol. 19, N. 3; Abril 1990, pp. 8-13. Reproducido con autorización. Traducción de Lucía Jones.

Agradecimientos: Por sus comentarios sobre borradores anteriores, le estoy agradecido a Michael Cole, Allan Collins, Marilyn Quinsaat y Chip Bruce. Earth Lab fue financiado por la National Science Foundation subvención MDR 8550449. La preparación de este artículo fue financiada por el Literacies Institute con una subvención de la Fundación Mellon al Education Development Center y al Center for Tecbnology in Education con la subvención número 1-135562167-A1 del Office of Educational Research and Improvement, U.S.A. Departamento de Educación, a Bank Street College of Education.

() De todos los artículos. Deberá solicitarse por escrito autorización de CL\&E y de los autores para el uso en forma de facsímil, fotocopia o cualquier otro medio de reproducción impresa. CL\&E se reserva el derecho de interponer las acciones legales necesarias en aquellos casos en que se contravenga la ley de derechos de autor. 